\title{
Erratum to: Information filtering by synchronous spikes in a neural population
}

\author{
Nahal Sharafi ${ }^{1} \cdot$ Jan Benda $^{4} \cdot$ Benjamin Lindner $^{2,3}$
}

Published online: 18 April 2015

(c) Springer Science+Business Media New York 2015

\section{Erratum to: J Comput Neurosci (2013) 34:285-301 DOI 10.1007/s10827-012-0421-9}

In Section 2.5 of our paper (Sharafi et al. 2013), we gave in Eq. (39) an approximate expression for the power spectrum of the synchronous output, consisting of a zeroth-order term and three correction terms, which are proportional to the signal's variance (our small parameter). One of these

The online version of the original article can be found at http://dx.doi.org/10.1007/s10827-012-0421-9.

Benjamin Lindner

benjamin.lindner@physik.hu-berlin.de

1 Max Planck Institute for Dynamics and Self-Organization, Göttingen, Germany

2 Bernstein Center for Computational Neuroscience Berlin, Philippstr. 13, Haus 2, 10115, Berlin, Germany

3 Department of Physics, Humboldt University Berlin, Newtonstr. 15, 12489, Berlin, Germany

4 Institute for Neurobiology, University Tübingen, Tübingen, Germany correction terms was miscalculated. The correct formula in Eq. (39) should read:

$$
\left.\begin{array}{rl}
S_{S O, S O} \simeq \alpha^{2}\left[\begin{array}{l}
\underbrace{S_{y_{0}, y_{0}} * \cdots * S_{y_{0}, y_{0}}(f)}_{\mathrm{n} \text { terms }} \\
+n\left(|\tilde{F} \chi|^{2} S_{S, s}\right) * \underbrace{S_{y_{0}, y_{0}} * \ldots * S_{y_{0}, y_{0}}(f)}_{(\mathrm{n}-1) \text { terms }}
\end{array}\right. \\
& +n(n-1) r_{0}^{2}\left(|\tilde{F} \chi|^{2} S_{s, s}\right) * \underbrace{S_{y_{0}, y_{0}} * \ldots * S_{y_{0}, y_{0}}(f)}_{(\mathrm{n}-2) \text { terms }}) \\
& +n(n-1) r_{0}^{2}\left\langle\hat{s}^{2}\right\rangle \underbrace{S_{y_{0}, y_{0}} * \ldots * S_{y_{0}, y_{0}}(f)}_{(\mathrm{n}-2) \text { terms }})
\end{array}\right]
$$

Note that we also replaced the phrase " $n$-times" etc. used in the original formula by the more precise formulation " $n$ terms".

Equation (39) in (Sharafi et al. 2013) was also used to approximate the power spectrum of the synchronous output for the special cases of populations with $n=2$ or $n=3$ Poisson neurons. Hence, the respective formulas for this power spectrum, Eqs. (51) and (52) in (Sharafi et al. 2013), have to be corrected as well. We recall that for Poisson neurons, we can simplify the general formula Eq. (39) using that $\chi(f)=r_{0}$ [Eq. (42) in (Sharafi et al. 2013)] and that $\left\langle\hat{s}^{2}\right\rangle_{s} \approx r_{0}^{2} D_{s} /(\sigma \sqrt{\pi})$ [Eq. (42) in (Sharafi et al. 2013)], assuming a high cutoff frequency of the stimulus $\left[f_{c} \gg 1 /(2 \pi \sigma)\right]$. 
The correct formula for $n=2$ replacing Eq. (51) in (Sharafi et al. 2013) reads:

$$
\begin{aligned}
& S_{S O, S O}(f)=\frac{r_{0}^{2} \alpha^{2}}{2}\left[4 r_{0}\left(1+4 D_{s} r_{0}\right) e^{-2 \beta f^{2}}+\sqrt{\frac{\pi}{\beta}} \times\right. \\
& \left.\left(1+2 r_{0} D_{s}\left[1+\operatorname{erf}\left(2 \sqrt{\beta}\left(f_{c}-f / 2\right)\right)\right]\right) e^{-\beta f^{2}}\right],
\end{aligned}
$$

where $\beta=2 \pi^{2} \sigma^{2}$ and we also used that erf $\left(\sqrt{\beta}\left(2 f_{c}+\right.\right.$ $f)) \approx 1$ for $f \in\left(0, f_{c}\right)$ which is valid for $f_{c} \gg 1 /(2 \pi \sigma)$ as already assumed above.

For $n=3$, Eq. (52) should be replaced by

$$
\begin{aligned}
& S_{S O, S O}(f)= \\
& \alpha^{2} r_{0}^{3}\left[3 r_{0}^{2}\left[1+D_{s}\left(6 r_{0}+2 \sqrt{\frac{2 \pi}{\beta}}\right)\right] e^{-2 \beta f^{2}}+\right. \\
& \frac{3 r_{0}}{2} \sqrt{\frac{\pi}{\beta}}\left(1+4 D_{s} r_{0}\left[1+\operatorname{erf}\left(2 \sqrt{\beta}\left(f_{c}-f / 2\right)\right)\right]\right) e^{-\beta f^{2}}+ \\
& \left.\frac{\pi / 2}{\sqrt{3} \beta}\left(1+3 D_{s} r_{0}\left[1+\operatorname{erf}\left(\sqrt{3 \beta}\left(f_{c}-f / 3\right)\right)\right]\right) e^{-\frac{2}{3} \beta f^{2}}\right] .
\end{aligned}
$$

We used the corrected formulas to recalculate our approximations for the power spectrum and coherence functions for the populations of Poisson neurons and of LIF neurons, respectively, and found virtually no difference to our previous results. It turned out that for the signal amplitudes used in our paper, the term in question only slightly affects the approximation of the power spectrum.

We also would like to emphasize that our qualitative explanation of information filtering by synchrony is based on the zeroth-order approximation of the power spectrum and thus is not affected at all by the modified correction term. Hence, all our conclusions drawn from the theory in (Sharafi et al. 2013) remain valid.

We would like to thank Alexandra Kruscha, Bernstein Center for Computational Neuroscience Berlin, for pointing out the error to us.

\section{References}

Sharafi, N., Benda, J., \& Lindner, B. (2013). Information filtering by synchronous spikes in a neural population. Journal of Computational Neuroscience, 34, 285. 